\title{
Paper
}

\section{Optimization for Greedy Non-maximum Suppression Based on Multi-task Convolutional Neural Network}

\author{
QINGQING HonG* ${ }^{* \dagger}$ Member, QIAN FAN ${ }^{\ddagger}$ Member \\ LIFENG ZHANG ${ }^{\dagger} \quad$ Member
}

(Received November 05, 2019, revised January 10, 2020)

\begin{abstract}
Non-maximum suppression (NMS) is an essential part of the face detection pipeline based on a convolutional neural network (CNN). The typical approach for NMS used by face detection is a greedy, locally optimal strategy, which is to localize objects from a set of candidate locations. However, NMS still has some shortcomings, such as sometimes the detection box has no relationship with a high classification score, which leads to misjudging face localization during NMS. In this paper, we observed that the NMS implemented on the multi-task convolutional neural network (MTCNN) is a cascaded network and the enhanced NMS based on MTCNN to achieve high performance during face detection and alignment. We employ WIDER FACE as the test dataset to evaluate our proposal. The precision and recall curves are drawn when using three subsets at different thresholds. The result shows that the proposed approach can perform better performance than traditional NMS.
\end{abstract}

Keywords: Face detection, face alignment, MTCNN, CNN, NMS.

\section{Introduction}

Face detection and alignment has been widely applied in computer vision to generate bounding boxes for identifying frontal face and assign them classification scores. However, the various appearance of faces imposed great challenges for tasks such as occlusions, large pose variation, and extreme lightings in practical applications.

Besides the challenge of various faces' appearance, few conventional approaches concentrated on the inherent correlation between face detection and alignment will also decrease the accuracy of face detector. Even if Chen et al.[1] presented a new state-of-art approach for face detection and alignment. The key proposal is to adopt random forest which take the differences of pixel value's differences into consideration. But the performance still limited by handcraft features. In this paper, we introduce a new framework represented by multi-task cascaded convolutional networks (MTCNN) to integrate face detection and alignment proposed by Kaipeng Zhang et al.[2]. The advantages of MTCNN is described as follows: First, MTCNN adopted a new cascade and lightweight CNN framework to combine face detection and alignment for achieving excellent realtime performance. Second, the online hard sample mining which is an efficient approach was proposed in order to improve the performance. Because original hard sample mining could decrease the performance due to offline manner.

\footnotetext{
* Corresponding: hongqingqing1221@gmail.com

${ }^{\dagger}$ Department of Electrical and Electronic Engineering, Kyushu Institute of Technology

1-1 Sensui-cho, Tobata-ku, Kitakyushu city, Fukuoka, Japan 804-8550

* School of Information Engineering, Yangzhou University

196 Huayang West road, Yangzhou, Province of Jiangsu, China
}

The detail of MTCNN we will describe in the next chapter. Although, MTCNN performs better consistently compared to other state-of-the-art techniques like P. Viola et al. [3] , H. Li et al.[4], Z. Zhang et al.[5] and Chen et al.[1].

However, there are still some shortcomings that exist in MTCNN. After classification and regression scores are generated by MTCNN, there is no constraint that can delete redundant bounding boxes and generate a specific box for one face. In order to avoid this problem, MTCNN employed greedy non-maximum suppression (NMS) as a postprocessing stage to obtain final detection boxes. However, the characteristics of greedy NMS is to make a hard decision and set a fixed threshold to decide the range of suppression. In this case, face misjudging will be occurred and the average precision of the entire network may be dropped.

To overcome the deficiency of greedy NMS, we will support a new improved NMS algorithm implemented on MTCNN. The improved NMS is to decay the scores of redundant boxes that have an overlap with the specific box as a consistent function.

The rest of this paper is organized as follow: In chapter 2, the related work that describes the detail of MTCNN and NMS. In chapter 3, specify the existing issue of NMS remained in MTCNN. In chapter 4, describe our research proposal. In chapter 5, describe our experiment and result.

\section{Related Work}

In this chapter, we will describe the detail of MTCNN proposed by Kaipeng Zhang et al.[2] and the greedy NMS algorithm.

2.1 MTCNN The overall frameworks of MTCNN is shown as Fig. 1 This framework performed a cascaded ar- 

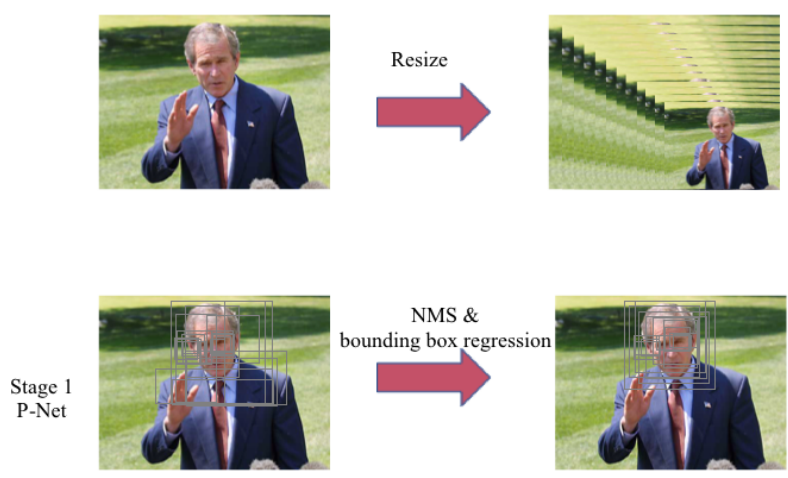

Input Image
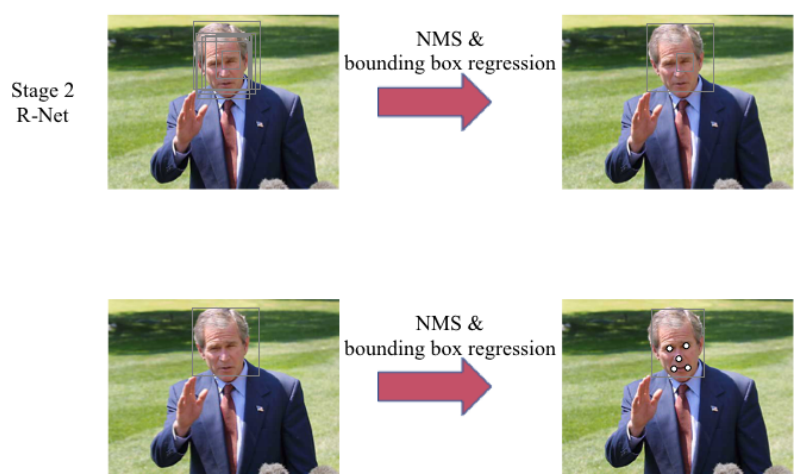

(a) $N=10$

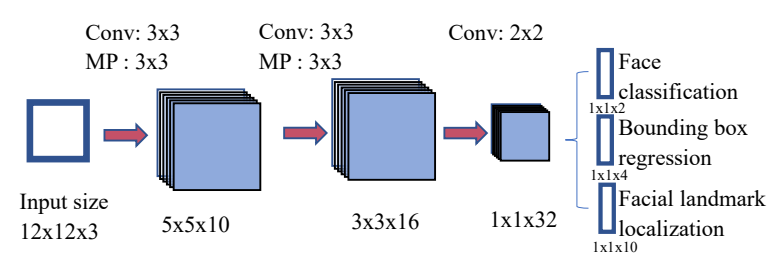

Figure 2: The architecture of P-Net

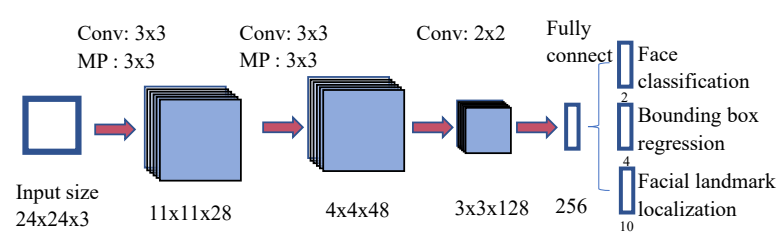

Figure 3: The architecture of R-Net

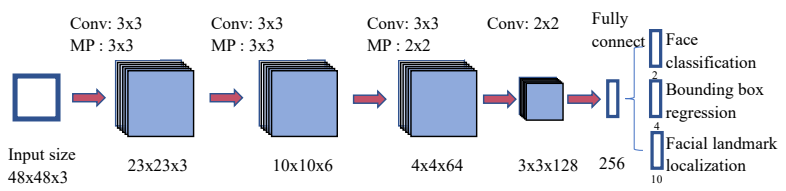

Figure 4: The architecture of O-Net

Figure 1: The overall framework of MTCNN $N$.

chitecture which consists of three stages network.

When images input into the framework, the framework will initially resize this image into different scales in order to establish a pyramid. And the result of the pyramid will be regarded as input for the three-stage network that we will describe in the follows:

(a) Proposal Network (P-Net):

In this stage, a fully convolutional network was implemented. And the framework adopted a similar approach proposed by S. S. Farfade et al.[6] to gain the candidate windows and bounding box regression vectors. Then, estimated bounding box regression vectors were used to calibrate the candidates. The bounding box regression is formulated as follows by employing Euclidean loss ( $\hat{y}_{i}^{\text {box }}$ indicate the regression target obtained from the network and $y_{i}^{b o x}$ is the ground truth):

$$
L_{i}^{b o x}=\left\|\hat{y}_{i}^{b o x}-y_{i}^{b o x}\right\|_{2}^{2}
$$

The last step is to employ NMS to merge the high overlapped candidates. The architecture of P-Net is shown as Fig. 2. Since face detection is a binary classification task, less filters should be needed but more discrimination. In this case that, from Fig. 2. we can observe that the number of filters has been reduced and the size of filter has been changed to $3 \times 3$.

(b) Refine Network (R-Net):

The second stage of the MTCNN is R-Net. The ba- sic architecture is convolutional neural network. Compared to the P-Net, a fully connected layer was added. After the input image processed by P-Net, many candidates windows still existed and then input to R-Net. Hence, large numbers of candidates will be rejected strictly by R-Net. The last step also the same as P-Net, calibrate candidates with bounding box regression and merge the candidates by using NMS. The architecture of R-Net is shown as Fig. 3.

(c) Output Network (O-Net)

The third stage of MTCNN is R-Net. Compared to the $\mathrm{R}-\mathrm{Net}$, the architecture of O-Net is more complexity. Since one more convolutional layer was added. The distinction between R-Net and O-Net is that the structure of O-Net will recognize the area of face through more supervision, and finally return five facial features. The architecture of O-Net is shown as Fig. 4.

2.2 NMS NMS is frequently used as an essential part of face detection which is post-processing step for removing the redundant candidate windows and obtains impressive effect shown as Fig. 5. We will use an example to show the result of NMS specifically. The Fig. 5a is the data set of detection boxes we defined by ourselves. The Fig. $5 b$ is the result after the NMS processed. We can find that in the middle area, the redundant boxes has been deleted. The NMS's principle is that search for local maximums and suppress non-maximum values.

The workflow of NMS is described as follows: first, 


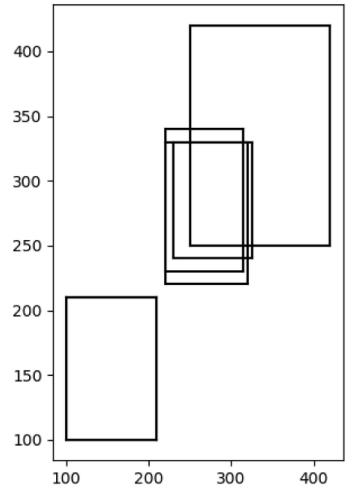

(a) The detection boxes we defined randomly

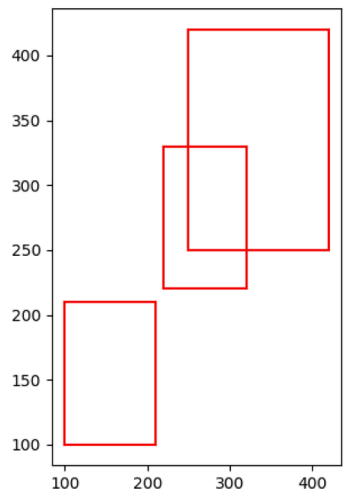

(b) The result after NMS processed
Figure 5: The process of NMS

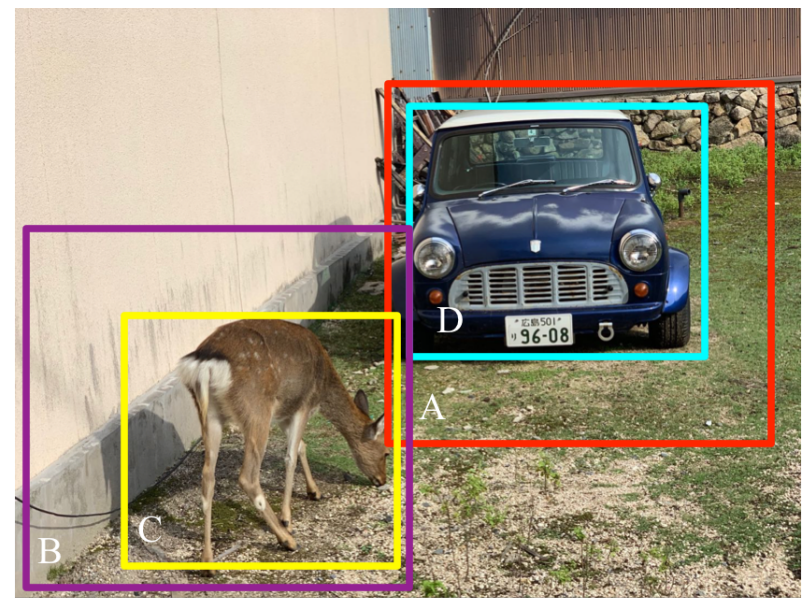

Score for 4 candidates

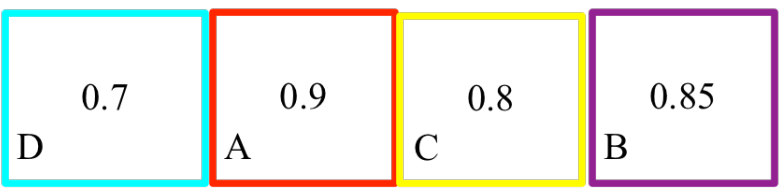

Figure 6: Specific the process of NMS

NMS starts with a list of detection boxes B which sorted by the confidence scores $\mathrm{S}$; Second, select the detection box which has maximum score $\mathrm{M}$ and add it into final detection set $\mathrm{D}$. Meanwhile, remove the box $\mathrm{M}$ which has the maximum from the list $\mathrm{B}$. Then we set a fixed threshold Nt and compute the remaining boxes' intersection-overunion (IOU) with box $\mathrm{M}$ in the list $\mathrm{B}$. And then remove the box from the list B which has greater IOU than threshold Nt. This process will be repeated for the remaining boxes in the list B. We will take an example to explain the process more vividly. As Fig. 6 shows, there exist four bounding boxes $\mathrm{A}, \mathrm{B}, \mathrm{C}, \mathrm{D}$. And sorted by confidence scores as $\mathrm{A}>\mathrm{B}>\mathrm{C}>\mathrm{D}$. Then mark bounding box $\mathrm{A}$ as the maximum score box. Compute the IOU that remaining box B, C, D overlap with box A. Make an assumption that D's IOU greater than the threshold we set in advance. Then we will remove box $\mathrm{D}$ from the candidates. After that, we select the box which has higher confidence score from B and C. And

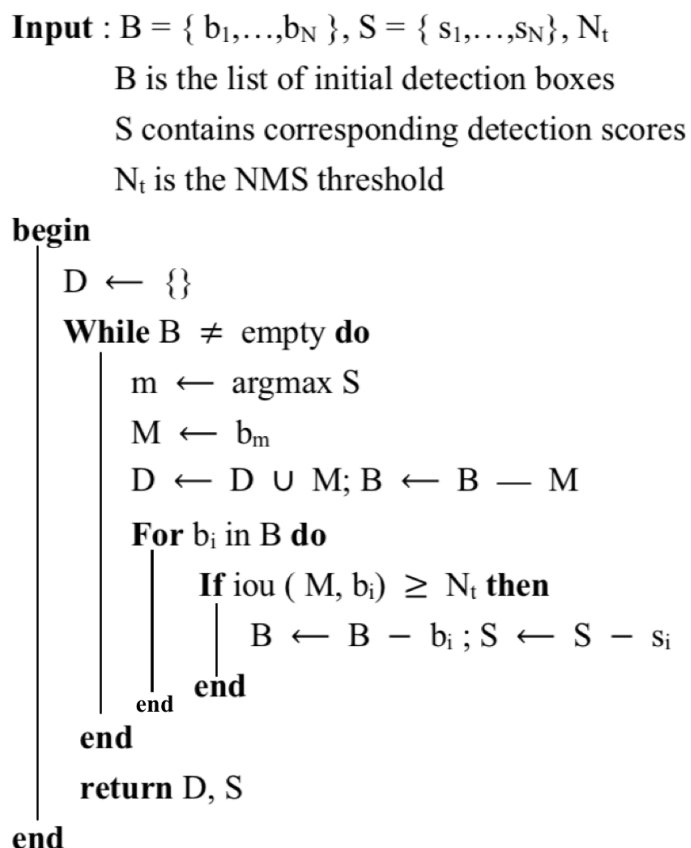

Figure 7: NMS removed the redundant candidate boxes.

compute the IOU between B and C. If IOU is greater than the threshold, the box which has the lower confidence score will be dropped.

The pseudocode also performed as following Fig. 7. In this case that, NMS shows good performance during face detection. However, due to its characteristics, NMS still has some limitations. We will describe it in the next section.

2.3 Pitfalls in traditional NMS As we mentioned in the previous chapter, NMS shows good performance implemented in the modern face detection techniques. But some pitfalls still exist in the NMS which will cause miss and false detection occurred. The major pitfall of NMS presented as follows:

(a) NMS set the score for adjacent detection box to zero forcibly which means remove the detection box whose IOU is greater than the threshold. In this case, if two or more objects are close to each other, all but one of the candidates will be removed, the result will be performed as Fig. 8(a).

(b) NMS will maintain the box with highest confidence score. However, some cases show that the neighboring boxes with a lower score may perform better for the true object like Fig. 8(b) shows.

(c) NMS will return the candidate boxes which are not removed. Meanwhile, many boxes with relatively lower confidence that haven't detected the true object will also be returned. This problem will be shown in Fig. 8(c). specifically.

Due to the pitfalls described previously, the traditional NMS will degrade the performance of MTCNN which take NMS as last step in each stage to merge candidate boxes. 


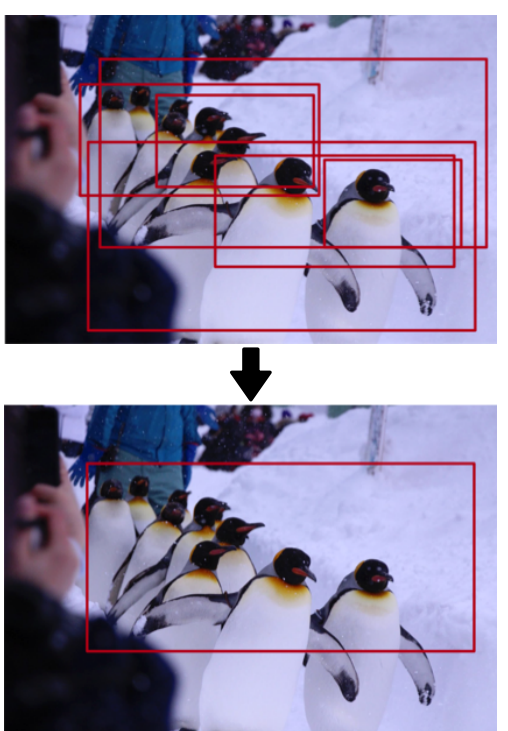

(a) The surrounding penguins will be ignored

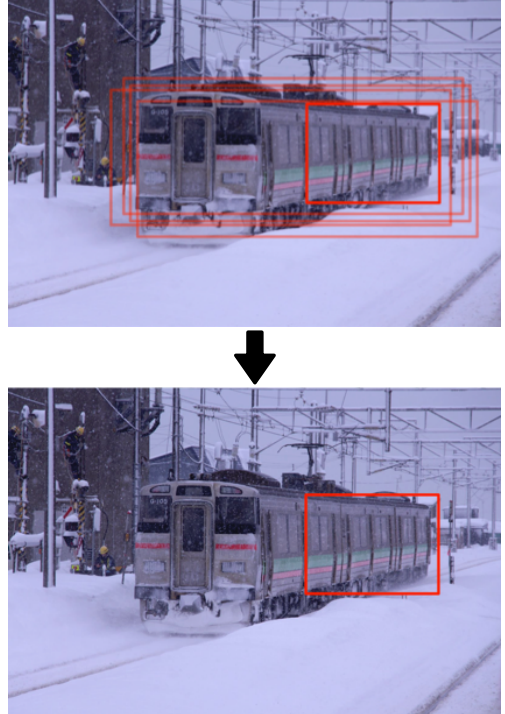

(b) The box with highest score may not be suitable

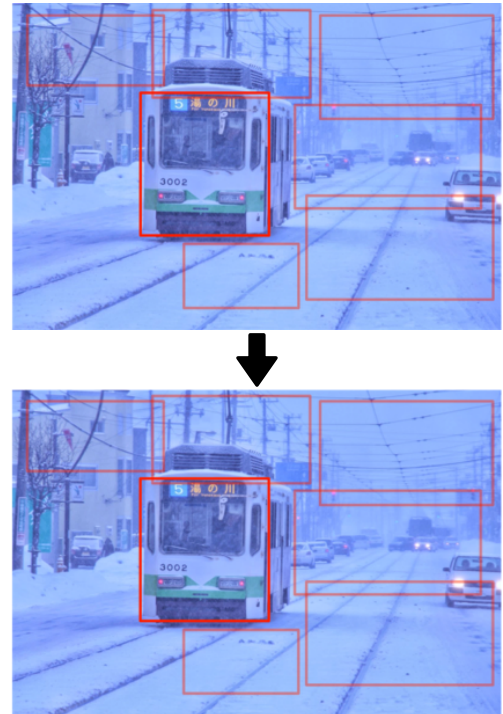

(c) Many false objects have been returned

Figure 8: Pitfalls exist in traditional NMS

In next section, we will adopt a new NMS algorithm proposed by Navaneeth Bodla et al.[8] for improving the performance of MTCNN consistently. The new NMS will decay the detection scores of all other neighboring boxed as a continuous function instead of setting the score to zero forcibly.

\section{Proposal}

Before we describe the new NMS, let's review the traditional NMS with a simple function presented as Eq. 2:

$$
s_{i}= \begin{cases}s_{i}, & \operatorname{IOU}\left(M, b_{i}\right)<N_{t} \\ 0, & \operatorname{IOU}\left(M, b_{i}\right) \geq N_{t}\end{cases}
$$

The parameter of $\mathrm{M}, b_{i}, N_{t}$, and $s_{i}$ have described in the previous chapter. Hence, we can simply find that NMS sets a fixed threshold $N_{t}$ which decides the candidate boxes which need to be removed or not. If some boxes' IOU smaller than the threshold, NMS will set the score to zero. To avoid the deficiency of traditional NMS, the new NMS adopts a linear function that decays the confidence score of neighbor boxes, which have overlaps with the highest confidence score box M. In fact, we should consider that the boxes have higher overlaps with $\mathrm{M}$ needed to be decayed more. Hence, Navaneeth Bodla et al.[8] proposed an equation presented as follows to achieve that the boxes which are far away from $\mathrm{M}$ will not be affected and boxes which are very close would be assigned a greater punishment.

$$
s_{i}=\left\{\begin{array}{cc}
s_{i}, & \operatorname{IOU}\left(M, b_{i}\right)<N_{t} \\
s_{i}\left(1-\operatorname{IOU}\left(M, b_{i}\right)\right), & \operatorname{IOU}\left(M, b_{i}\right) \geq N_{t}
\end{array}\right.
$$

From the Eq. 3, we should consider another problem that the linear function is not continuous. When the IOU exceeds the overlap threshold $N_{t}$, mutation will be occurred.

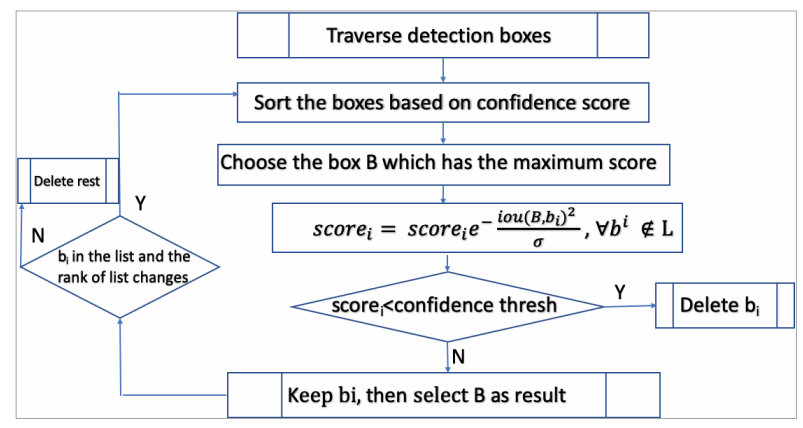

Figure 9: The workflow of NMS gaussian based

In this case that, a large changes of the detections' rank list will be happed. For taking this problem into consideration, another function which can be continuously decay the confidence score should be designed. The function should achieve the following conditions:

(a) Boxes with lower IOU which means far away from M, the penalty should be increased gradually.

(b) Boxes with higher overlaps with $\mathrm{M}$, the penalty should be increased significantly.

Based on the conditions above and mutation occurred in previous linear function, Gaussian function has been adopted. The formula presented as Eq. 4.

$$
s_{i}=s_{i} e^{-\frac{\operatorname{IOU}\left(M, b_{i}\right)^{2}}{\sigma}}, \forall b_{i} \notin D
$$

Where $\sigma$ represents the weight of Gaussian Function. Each iteration should apply for this function and update the remaining boxes' score. Fig. 9 shows the workflow of gaussian based NMS. It can be described as follow: When 
the detection process finished, then traverse the detection boxes. After traversing, we will sort the boxes based on the confidence score as a list. Then select box B which has the maximum score from the list. Calculate the IOU between rest boxes and $\mathrm{B}$. And then, update the confidence score of rest boxes based on Eq. 4. Compare each box's confidence score with confidence threshold. If the confidence score is smaller than the threshold, delete the box from the list. Oppositely, keep the box and remove B from the list. And select the $\mathrm{B}$ as a result. And then keep doing the iteration until no boxes in the list then this iteration will be stopped.

Next chapter, we will employ the improved NMS into MTCNN to identify the improvement of original framework used by traditional NMS. Our purpose is to achieve higher average precision and shorten the entire detection time.

\section{Experiment}

4.1 Environment We performed our experiments for identifying our proposal that shows good performance compared to traditional NMS. The WIDER FACE would be adopted to perform during the experiment which is a face detection benchmark dataset. The WIDER FACE chooses 32203 images and label 393703 faces with a high degree of variability in scale, pose and occlusion as depicted in the sample images. For each event, 40\%, 10\% and 50\% are selected as training, validation and testing sets. In this work, we employed the validation set as our test dataset. The experiment implemented on the Deeplearning-Box with 3 pieces of GeForceGTX 1080Ti 11GB HDMI and 64GB main memory.

4.2 Measure metrics In order to compare the result with traditional NMS implemented on MTCNN. The improvement of recall and precision was calculated. The recall means the ratio of the number correctly detected to the number of all faces in the test set. The equation will be shown as Eq. 5.

$$
\text { Recall }=\frac{t p}{t p+f n}
$$

Where the $t p$ represents true positives which mean faces detected as positive by MTCNN is positive (meaning they are correct), and $f n$ represents false negatives which mean faces that MTCNN detected as negative but are positive (incorrect). Denominator of Eq. 5 represent that how many images with faces exist in the test set.

Another metric is precision. Precision represent that the ratio of true positive in the detection images as Eq. 6 showed:

$$
\text { Precision }=\frac{t p}{t p+f p}=\frac{t p}{n}
$$

The $f p$ means false positives which means faces that MTCNN incorrectly detected as positives that are negative. The sum of $f p$ and $t p$ is $n$ represents the total number of images which has been detected. Based on these two metrics, we specify the improvement of our proposal specifically.

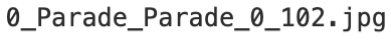

Figure 10: Format of generated txt file.

4.3 Result analysis In this section, we will describe our detail of the experiment and analyze the proposed results compared to the traditional algorithm. We designed a program that can produce a texture file for each detecting box. In this file, the format formed by image name and number, the number of faces exist in the image and coordinate shown as Fig. 10. Then the result will be computed with "wider face val.txt" which supported by the official organization of WIDER FACE to calculate the precision and recall. The "wider face val.txt" is used to be recorded the true positive information.

In experiment, in order to show significant improvement, we utilized the second stage of MTCNN, which is R-Net to evaluate our proposal. Since we have set the $\sigma$ from 0.1 to 0.7 to test the performance of optimized NMS on coco dataset, and we find that at the beginning, the average precision increased gradually until it reached 0.5 . And From the value of $\sigma$ increased from 0.6 , the average precision decreased gradually. Take this into consideration, the weight $\sigma$ of proposed gaussian NMS was set to 0.5. And the threshold Nt for each stage of MTCNN as [0.5, 0.6, $0.6]$, this parameter was set by MTCNN originally, we will adopt these three thresholds in order to compare with the optimized NMS. The result will be shown below Fig. 11.

In Fig. 11, we show the PR curve and average precision with three subsets: Easy, Hard, Medium. The hard set is a superset of easy and medium, which means contain all faces taller than 10 pixels, the performance on hard set can represent the performance on the entire test more accurately. From Fig. 11, we specify that with the recall increased, the precision will be decreased. Because if recall becomes high, the number of boxes will become more. In this case, the precision will be decreased. Our result shows that our proposal can achieve a high performance based on MTCNN. Meanwhile, we set a different threshold for calculating the PR curve. From the result of PR curve, we can see among the three subsets with different thresholds. Our proposed NMS can achieve better results than traditional NMS. The average precision was improved by $11.7 \% \sim 15.8 \%$.

Considering the result of Fig. 11, two test images were selected from WIDER FACE dataset. The detection result shows that the detection boxes were suppressed more and the precision also increased. We also employ proposed NMS with Gaussian function to compare to traditional NMS. The effect pictures are shown as Fig. 12. 

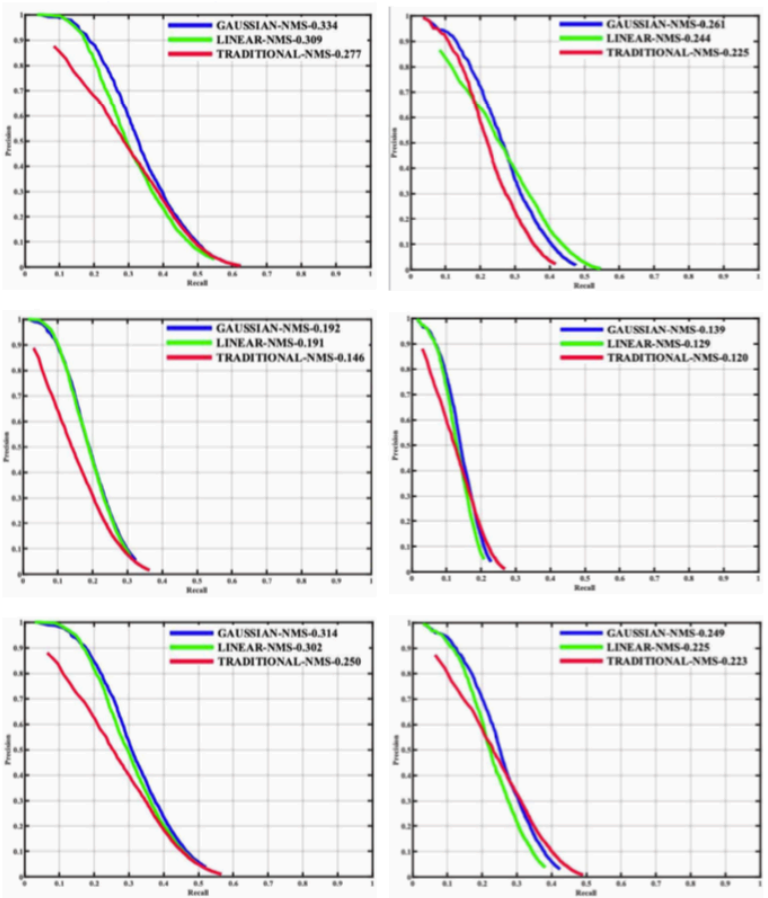

(a) Precision and and Medium

Figure 11: Precision vs Recall produced by three functions based on MTCNN.

\section{Conclusions}

This paper adopted a new NMS algorithm to improve the performance of MTCNN. The proposed NMS is to decay the confidence score continuously instead of setting the sore to zero forcibly, which is the principle of traditional NMS.

In the experiment, WIDER FACE dataset was used to identify the improvement of proposed implementation on MTCNN. And calculate the recall and precision at different thresholds. The new NMS algorithm shows a good result compared to traditional NMS. From the resulting images, the detection boxes are significantly decreased after applying new NMS, and the average precision also achieved good improvement from $11.7 \%$ to $15.8 \%$. However, from these two results, we can find that there still several faces that can' $\mathrm{t}$ be detected. And the average precision on hard set is a bit worse since proposed NMS based on MTCNN focused on learning easy faces.

Therefore, further research on hard face detection will be made to improve the performance of MTCNN by decreasing the number of faces that can' $t$ be detected.

\section{References}

[1] D. Chen, S. Ren, Y. Wei, X. Cao and J. Sun, "Joint cascade face detection and alignment", European Conference on Computer Vision (ECCV 2014), Vol.8694, pp.109-122, 2014. DOI: $10.1007 / 978-3-319-10599-4 \_8$

[2] K. Zhang, Z. Zhang, Z. Li and Y. Qiao, "Joint Face Detection and Alignment using Multi-task Cascade Convolutional

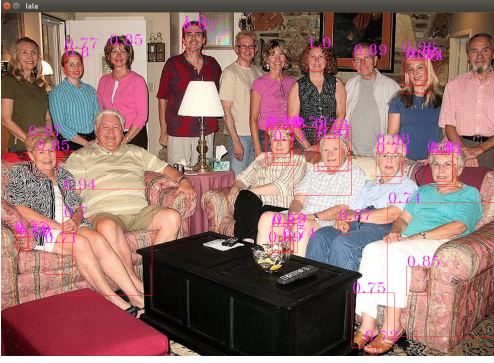

(a) Traditional NMS

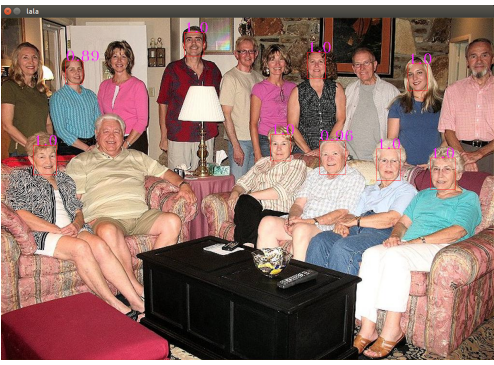

(b) Proposed NMS(Gaussian Function)

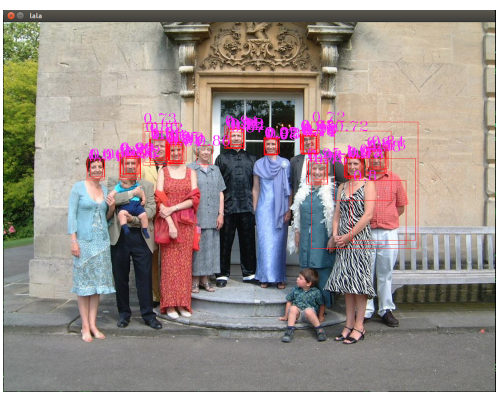

(c) Traditional NMS

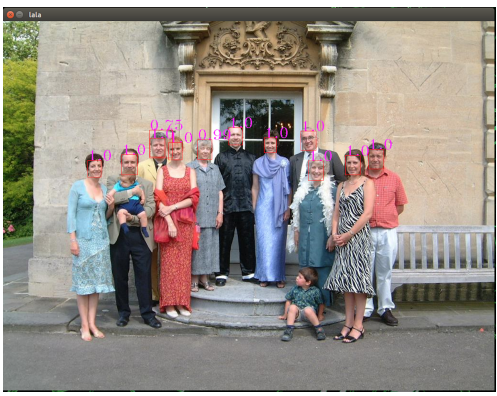

(d) Proposed NMS(Gaussian Function)

Figure 12: Comparison of original NMS and proposed NMS

Networks", IEEE Signal Processing Letters, Vol.23, No.10, pp.1499-1503, 2016. DOI: 10.1109/LSP.2016.2603342

[3] P. Viola and M. J. Jones, "Robust real-time face detection", International Journal of Computer Vision, Vol.57, No.2, pp.137-154, 2004. DOI: 10.1023/B:VISI.0000013087.49260.fb

[4] H. Li, Z. Lin, X. Shen, J. Brandt and G. Hua, "A convolutional neural network cascade for face detection", 2015 IEEE Conference on Computer Vision and Pattern Recognition (CVPR 2015), pp.5325-5334, 2015. DOI: 10.1109/CVPR.2015.7299170

[5] Z. Zhang, P. Luo, C. C. Loy and X. Tang, "Facial landmark detection by deep multi-task learning", European Confer- 
ence on Computer Vision (ECCV 2014), Vol.8694, pp.94108, 2014. DOI: 10.1007/978-3-319-10599-4_7

[6] S. S. Farfade, M. J. Saberian and L. J. Li, "Multi-view face detection using deep convolutional neural network", Proceedings of the 5th ACM on International Conference on Multimedia Retrieval (ICMR 2015), pp.643-650, 2015. DOI: 10.1145/2671188.2749408

[7] R. Rothe, M. Guillaumin and L. V. Gool, "Non-Maximum Suppression for Object Detection by Passing Messages between Windows", Asian Conference on Computer Vision (ACCV 2014), Vol.9003, pp.290-306, 2014. DOI: 10.1007/978-3-319-16865-4 19

[8] N. Bodla, B. Singh, R. Chellappa and L. S Davis, "Soft-NMS-Improving Object Detection With One Line of Code", The IEEE International Conference on Computer Vision (ICCV 2017), pp.5561-5569, 2017. DOI: 10.1109/ICCV.2017.593

[9] K. He, G. Gkioxari, P. Dollar and R. Girshick, "Mask rcnn", The IEEE International Conference on Computer Vision (ICCV 2017), Vol.42, No.2, pp.2961-2969, 2017. DOI: 10.1109/TPAMI.2018.2844175

[10] J. Hosang, R. Benenson and B. Schiele, "Learning non-maximum suppression", 2017 IEEE Conference on Computer Vision and Pattern Recognition (CVPR 2017), pp.4507-4515, 2017. DOI: 10.1109/CVPR.2017.685

[11] W. Liu, D. Anguelov, D. Erhan, C. Szegedy, S. Reed, C. Y. Fu and A. C. Berg, "SSD: Single shot multibox detector", European conference on computer vision(ECCV 2016), Vol.9905, pp.21-37, 2016. DOI: 10.1007/978-3-319-46448$0 \_2$

[12] Y. Rao, D. Lin, J. Lu and J. Zhou, "Learning Globally Optimized Object Detector via Policy Gradient", 2018 IEEE Conference on Computer Vision and Pattern Recognition (CVPR 2018), pp.6190-6198, 2018. DOI: 10.1109/CVPR.2018.00648

[13] Q. Zhu, M. C. Yeh, K. T. Cheng and S. Avidan, "Fast human detection using a cascade of histograms of oriented gradients", 2006 IEEE Computer Society Conference on Computer Vision and Pattern Recognition (CVPR 2006), pp.1491-1498, 2006. DOI: 10.1109/CVPR.2006.119

[14] C. Zhang and Z. Zhang, "Improving multiview face detection with multi-task deep convolutional neural network", IEEE Winter Conference on Applications of Computer Vision, pp.1036-1041, 2014. DOI: 10.1109/WACV.2014.6835990

[15] Z. Zhang, P. Luo, C. C. Loy and X. Tang, "Facial Landmark Detection by Deep Multi-task Learning", European conference on computer vision(ECCV 2014), Vol.8694, pp.94-108, 2014. DOI: 10.1007/978-3-319-10599-4 7

[16] B. Yang, J. Yan, Z. Lei and S. Z. Li, "Convolutional Channel Features”, 2015 IEEE International Conference on Computer Vision(ICCV 2015), pp.82-90, 2015. DOI: 10.1109/ICCV.2015.18

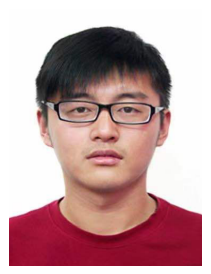

Qingqing Hong (Member) received the B.S. degree in Kunming University of Science and Technology in 2013. He received Master of Engineering from Waseda University, Japan in 2017. Since 2017, he has been a Ph.D. candidate in Electrical Engineering and Electronics from Kyushu Institute of Technology, Japan. His current research interests include machine learning, deep learning and object detections.

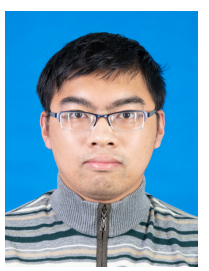

Qian Fan (Member) received the B.S. degree in Electronic Information Engineering from Soochow University in 2010. He received Master of Engineering from Kyushu Institute of Technology, Japan and from Yangzhou University, China in 2014. He received Ph.D. degrees in Electrical Engineering and Electronics from Kyushu Institute of Technology, Japan in 2017. Since 2018, he has been a lecturer in Department of Electronic Communication Engineering, College of Information Engineering, Yangzhou University, China. His current research interests include sensor application systems, computer vision and image processing.

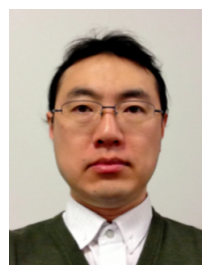

Lifeng Zhang (Member) received the B.S. degree in Electronic Engineering from Southeast University in 1994. He received M.S. and Ph.D. degrees in Electrical Engineering from Kyushu Institute of Technology, in 1999 and 2001, respectively. From 2002 to 2013, he was an assistant Professor at the Kyushu Institute of Technology. Since 2013, he has been an associate professor at Kyushu Institute of Technology. His current research interests include computer vision, image processing, biometrics authentication and remote sensing system. He is a member of IIAE, IEEE, and IEICE. 